\title{
On the Compatibility of Ground-based and Space-based Data: WASP-96 b, an Example*
}

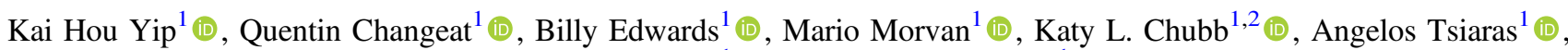 \\ Ingo P. Waldmann ${ }^{1}$ (D), and Giovanna Tinetti ${ }^{1}$ (i) \\ ${ }^{1}$ Department of Physics and Astronomy, University College London, Gower Street, WC1E 6BT London, UK; kai.yip.13@ucl.ac.uk \\ ${ }^{2}$ SRON Netherlands Institute for Space Research, Sorbonnelaan 2, 3584 CA, Utrecht, The Netherlands \\ Received 2020 June 25; revised 2020 September 11; accepted 2020 October 13; published 2020 December 2
}

\begin{abstract}
The study of exoplanetary atmospheres relies on detecting minute changes in the transit depth at different wavelengths. To date, a number of ground- and space-based instruments have been used to obtain transmission spectra of exoplanets in different spectral bands. One common practice is to combine observations from different instruments in order to achieve a broader wavelength coverage. We present here two inconsistent observations of WASP-96 b, one by the Hubble Space Telescope (HST) and the other by the Very Large Telescope (VLT). We present two key findings in our investigation: (1) a strong water signature is detected via the HST WFC3 observations and (2) a notable offset in transit depth $(>1100 \mathrm{ppm})$ can be seen when the ground-based and space-based observations are combined. The discrepancy raises the question of whether observations from different instruments could indeed be combined. We attempt to align the observations by including an additional parameter in our retrieval studies but are unable to definitively ascertain that the aligned observations are indeed compatible. The case of WASP-96 b signals that compatibility of instruments should not be assumed. While wavelength overlaps between instruments can help, it should be noted that combining data sets remains risky business. The difficulty of combining observations also strengthens the need for next-generation instruments that possess broader spectral coverage.
\end{abstract}

Unified Astronomy Thesaurus concepts: Exoplanet atmospheres (487); Exoplanet atmospheric composition (2021); Observational astronomy (1145); Bayesian statistics (1900)

\section{Introduction}

The field of exoplanetary science is rapidly expanding, with the discovery of new planets on a weekly basis becoming commonplace. There is growing interest in gaining a deeper understanding of these worlds and their atmospheric structure. Pioneering works by numerous teams have detected molecular species, alkali metals, and other carbon-bearing species present in the exoplanetary atmosphere (e.g., Charbonneau et al. 2002; Vidal-Madjar et al. 2004; Tinetti et al. 2007; Barman 2008; Redfield et al. 2008; Swain et al. 2009; Fossati et al. 2010; Linsky et al. 2010). Although many of the pioneering works have been done using space-based instruments due to the absence of atmosphere, in recent years ground-based instruments have also made significant contributions to our understanding of exoplanetary atmospheres (e.g., Barman et al. 2015; Macintosh et al. 2015; Gravity Collaboration et al. 2019; Ehrenreich et al. 2020; Merritt et al. 2020; Bourrier et al. 2020).

The installation of Wide-Field-Camera 3 (WFC3) on board the Hubble Space Telescope (HST) has further enriched our understanding of these remote worlds. The introduction of the scanning mode was pivotal in providing high-confidence detection of numerous molecular species such as $\mathrm{H}_{2} \mathrm{O}$ (e.g., Mandell et al. 2013; Ehrenreich et al. 2014; Wakeford et al. 2018; Tsiaras et al. 2019; Mikal-Evans et al. 2020; Pluriel et al. 2020b), $\mathrm{NH}_{3}$ (MacDonald \& Madhusudhan 2017) and $\mathrm{TiO}$ (Haynes et al. 2015; Edwards et al. 2020a). Over the past decade HST WFC3, and other instruments such as Spitzer IRAC and HST STIS, have observed tens of exoplanets and the rich amount of spectral data has led to initial population studies between atmospheres of different exoplanets (e.g., Iyer et al. 2016;

\footnotetext{
* These authors contributed equally to this work.
}

Sing et al. 2016; Fisher \& Heng 2018; Tsiaras et al. 2018; Pinhas et al. 2019).

WASP-96 b is a transiting gaseous hot Jupiter discovered by Hellier et al. (2014) during the WASP-South Survey. It orbits around a G8 star with a V magnitude of 12.2. Table 1 summarizes the stellar and planetary parameters of the WASP96 system. WASP-96 b was observed previously with Nikolov et al. (2018) using the FORS2 spectrograph on the Very Large Telescope (VLT). They obtained an optical transmission spectrum spanning from 0.35 to $0.80 \mu \mathrm{m}$. Their analysis showed that the atmosphere of the planet is cloud-free, under the assumption of chemical equilibrium, and measured an absolute sodium abundance of $\log \varepsilon_{\mathrm{Na}}=6.9_{-0.4}^{+0.6}$ based on the strong sodium profile in the optical wave band. The temperature of the atmosphere was found to be at $T=1710_{-200}^{+150} \mathrm{~K}$, which is notably higher than the equilibrium temperature of the planet, $T_{\text {eq }}=1285 \pm 40 \mathrm{~K}$.

In this work we present the HST transmission spectrum of WASP-96 b, obtained with both the G102 $(0.8-1.1 \mu \mathrm{m})$ and G141 $(1.1-1.7 \mu \mathrm{m})$ grisms. Our atmospheric retrieval of this data uncovers a strong water signature. To achieve a more comprehensive analysis of the planetary atmosphere, we attempt to combine this with the data from Nikolov et al. (2018). However, we find a large offset between the groundbased and space-based data sets. We explore a method of correcting for this issue when there is wavelength overlap and explore the risks associated with combining data sets that cannot be verified to be compatible in absolute transit depth.

\section{Data Analysis and Atmospheric Modeling}

\subsection{HST Data Reduction}

The HST data of WASP-96 b were acquired by proposal 15469 led by Nikolay Nikolov and were taken in December 
Table 1

Details of the WASP-96 System Used in This Study

\begin{tabular}{lc}
\hline \hline Parameters & Value \\
\hline$R_{s}\left[R_{\oplus}\right]$ & $1.05 \pm 0.05^{*}$ \\
$M_{s}\left[\mathrm{M}_{\oplus}\right]$ & $1.06 \pm 0.09^{*}$ \\
$T_{s}[\mathrm{~K}]$ & $5540 \pm 140$ \\
$M_{p}\left[\mathrm{M}_{\text {Jup }}\right]$ & $0.48 \pm 0.03^{*}$ \\
$R_{p}\left[R_{\text {Jup }}\right]$ & $1.20 \pm 0.06^{*}$ \\
$\mathrm{~T}_{\text {Eff }}(\mathrm{K})$ & $1285 \pm 40^{*}$ \\
$\mathrm{a} / R_{s}$ & $8.84 \pm 0.1^{\dagger}$ \\
$i[$ deg $]$ & $85.14 \pm 0.2^{\dagger}$ \\
$P_{\text {orb }}[$ days $]$ & $3.4252602 \pm 0.0000027^{*}$ \\
$T_{\text {mid }}[\mathrm{BJD}$ TDB $]$ & $2456258.062876 \pm 0.0002^{*}$ \\
${ }^{*}$ Hellier et al. (2014) & ${ }^{\dagger}$ Nikolov et al. (2018) \\
\hline
\end{tabular}

2018. We obtained the raw spatially scanned spectroscopic images from the Mikulski Archive for Space Telescopes ${ }^{3}$ and used Iraclis, ${ }^{4}$ a specialized, open-source software for the analysis of WFC3 scanning observations (Tsiaras et al. 2016b). The reduction process included the following steps: zero-read subtraction, reference pixels correction, nonlinearity correction, dark current subtraction, gain conversion, sky background subtraction, calibration, flat-field correction, and corrections for bad pixels and cosmic rays. For a detailed description of these steps, we refer the reader to Tsiaras et al. (2016b).

The reduced spatially scanned spectroscopic images were then used to extract the white and spectral light curves. We then discarded the first orbit of the visit as it presents stronger wavelength-dependent ramps. For the fitting of the white light curves, the only free parameters were the midtransit time and planet-to-star ratio. We did not fit for the inclination or reduced semimajor axis, as ingress and egress were not observed in each data set. The limb-darkening coefficients were selected from using the models of Claret et al. $(2012,2013)$ and using the stellar parameters from Hellier et al. (2014). The fitted white and spectral light curves for the G102 and G141 transmission observations are shown in Figures 1 and 2.

\subsection{Spitzer IRAC Data Reduction}

Additionally, two transits of WASP-96 b had been observed with Spitzer IRAC (program ID 14255). We used the Transit Light Curve Detrending Long Short-Term Memory (TLCDLSTM) pipeline from Morvan et al. (2020) to detrend and fit the Spitzer data from the outer transit and centroids movements. The architecture is the same as in the aforementioned study except for the number of hidden units reduced to 64 and dropout rate set to 0.1 in order to prevent over fitting on the out-of-transit. Figure 3 shows the detrended light curves and the best-fit model to the data. For both data sets, the only free transit parameters were the planet-to-star radius ratio and the transit midtime, with the other model parameters fixed to those in Table 1.

We also fitted the detrended light curve while allowing the planet semimajor axis to star radius ratio $\left(\mathrm{a} / R_{\mathrm{s}}\right)$ and inclination (i) to vary. For both channels, the retrieved values for the transit depth and epoch remain very close to the ones found with the two orbital parameters fixed to the values from Nikolov et al. (2018). Furthermore, values retrieved for the semimajor axis

\footnotetext{
3 https://archive.stsci.edu/hst/

4 https://github.com/ucl-exoplanets/Iraclis
}
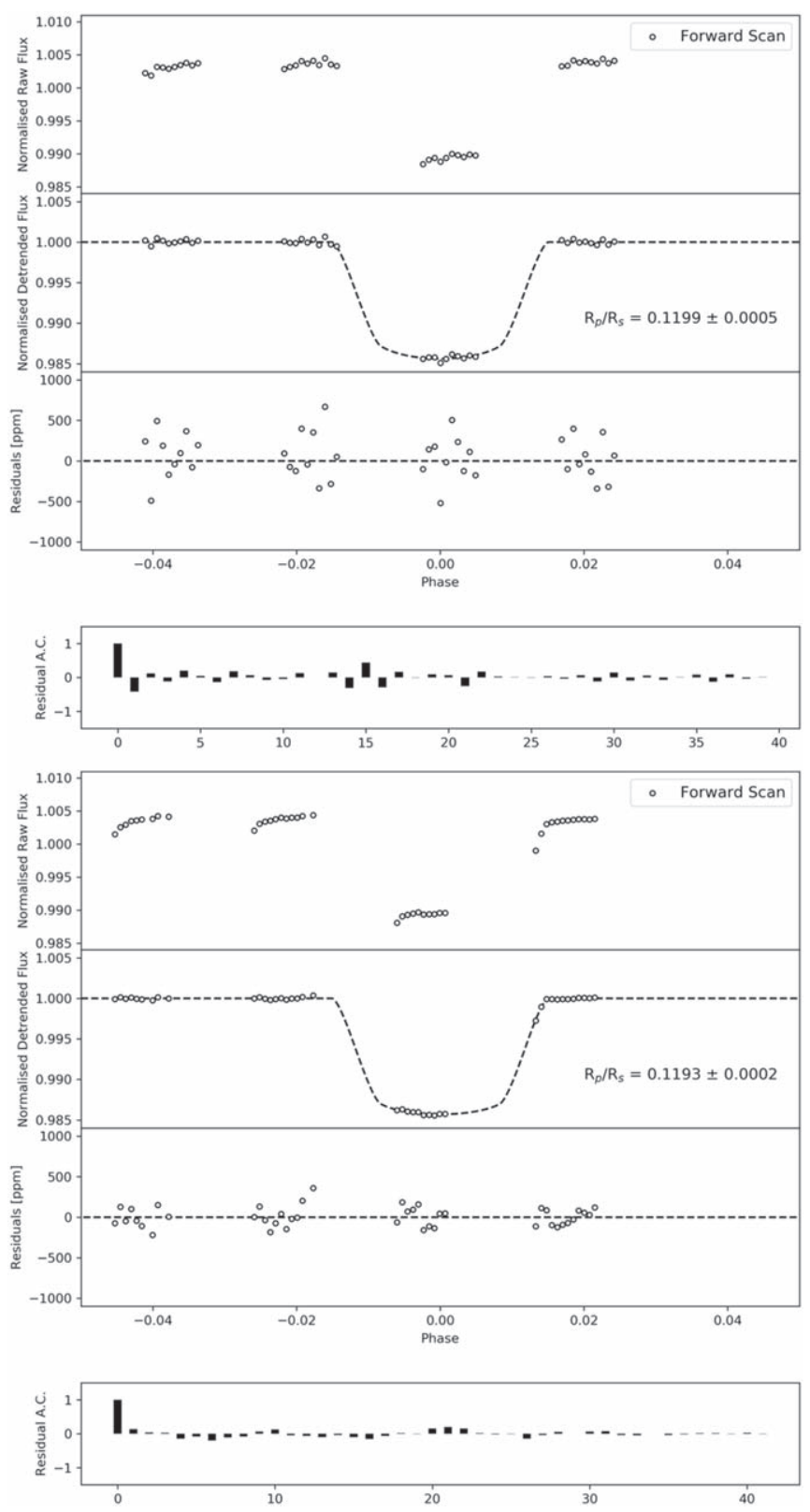

Figure 1. White light curves for the G102 (top) and G141 (bottom) transit observations of WASP-96 b. First panel: raw light curve, after normalization. Second panel: light curve, divided by the best-fit model for the systematics. Third panel: residuals for best-fit model. Fourth panel: autocorrelation function of the residuals.

and inclination shown in Table 2 are compatible with those from Nikolov et al. (2018) to $1 \sigma$.

\subsection{TESS Data Reduction}

Keeping exoplanet transit ephemeris fresh is crucial for allowing further atmospheric characterization. Here, we use our Hubble observations along with data from Spitzer and the Transiting Exoplanet Survey Satellite (TESS, Ricker et al. (2014)) to update the orbital period and transit epoch of WASP-96 b. TESS data are publicly available through the MAST archive and we follow the procedure from Edwards et al. (2020b) to download, clean, and fit the 2 minute cadence Pre-search Data Conditioning (PDC) light curves (Smith et al. 2012; Stumpe et al. 2012, 2014). WASP-96 b was observed 

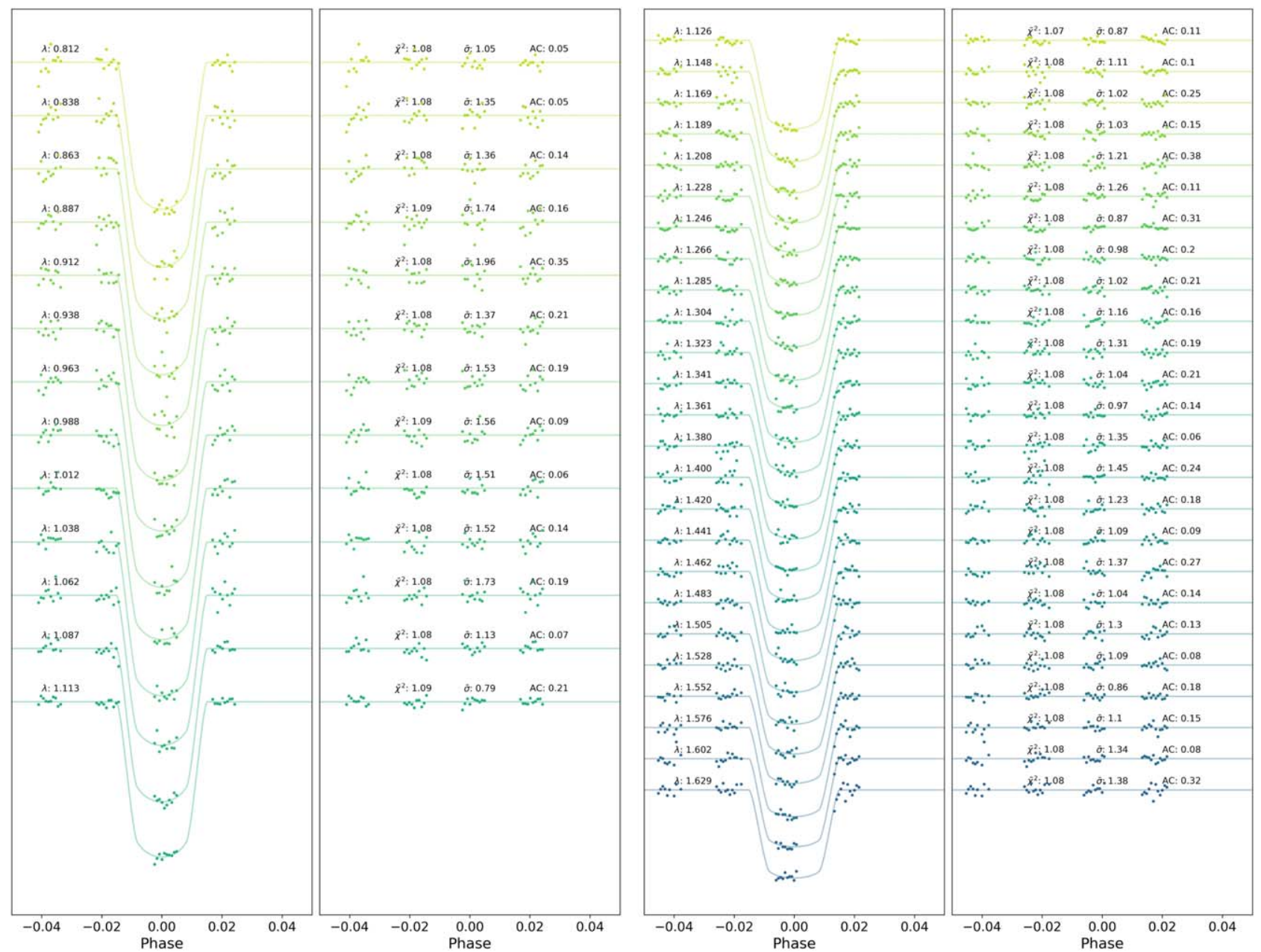

Figure 2. Spectral light-curve fits from Iraclis for the G102 (left) and G141 (right) transmission spectra where, for clarity, an offset has been applied. In each plot, the panels have the following definitions. Left panel: the detrended spectral light curves with the best-fit model plotted. Right panel: residuals from the fitting with values for the chi-squared $\left(\chi^{2}\right)$, the standard deviation with respect to the photon noise $(\bar{\sigma})$, and the autocorrelation (AC).

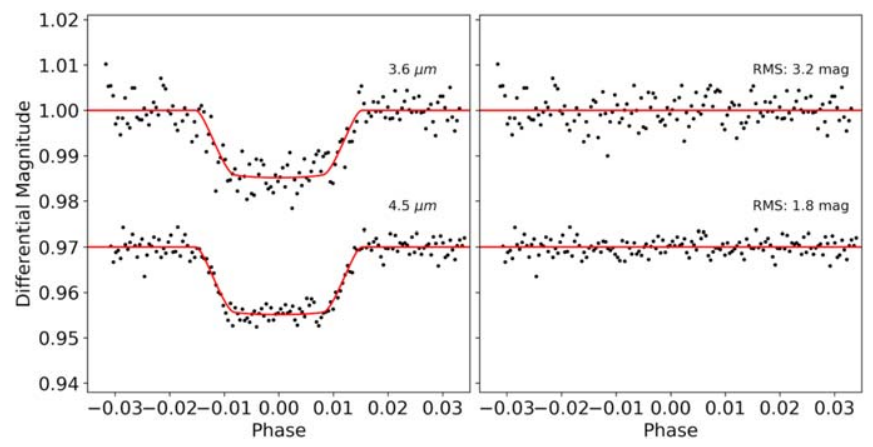

Figure 3. Fitted Spitzer transits of WASP-96 b for each IRAC channel. Left: detrended light curves and best-fit model. Right: residuals from the fitting.

Table 2

Comparison of Orbital Parameters between Different Data Sets

\begin{tabular}{lcccc}
\hline \hline Parameter & Nikolov et al. (2018) & TESS & IRAC C1 & IRAC C2 \\
\hline $\mathrm{a} / R_{\mathrm{s}}$ & $8.84 \pm 0.1$ & $8.85_{-0.10}^{+0.62}$ & $8.66_{-0.12}^{+0.14}$ & $8.78_{-0.06}^{+0.06}$ \\
$\mathrm{i}[\mathrm{deg}]$ & $85.14 \pm 0.2$ & $85.55_{-0.34}^{+0.39}$ & $85.36_{-0.13}^{+0.14}$ & $85.21_{-0.06}^{+0.07}$ \\
\hline
\end{tabular}

during Sector 2, and after excluding bad data, seven transits were recovered. These were individually fit and are shown in Figure 4. In our main analysis, the only free parameters were

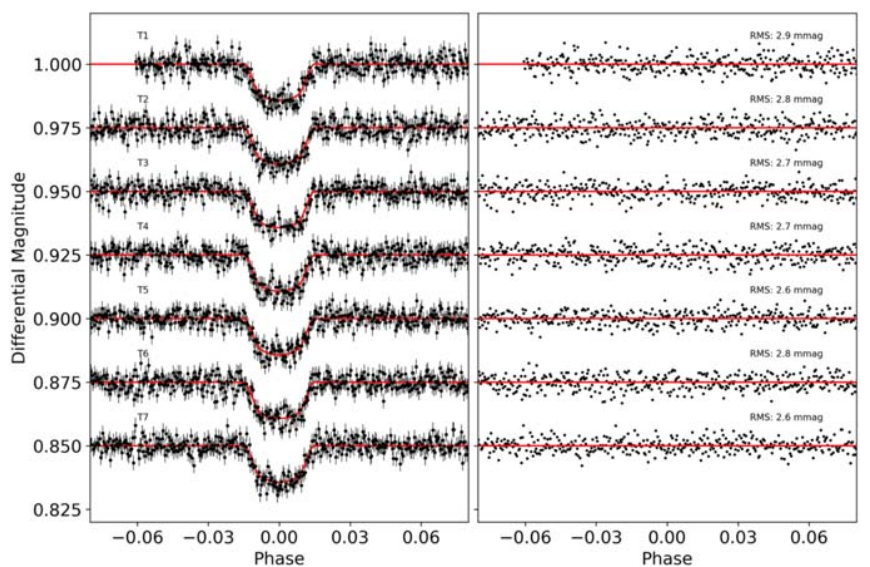

Figure 4. Fitted TESS transits of WASP-96 b. Left: detrended light curves and the best-fit model. Right: residuals from the fitting.

the transit midtime and the planet-to-star radius ratio. However, we also performed a fitting of all the TESS data where further orbital parameters, namely the inclination (i) and planet semimajor axis to star radius ratio $\left(\mathrm{a} / R_{\mathrm{s}}\right)$ were allowed to vary. The results are summarized in Table 2 along with similar fits for the Spitzer data. 
Table 3

List of the Retrieved Parameters, Their Uniform Prior Bounds and the Scaling Used

\begin{tabular}{lccc}
\hline \hline & \multicolumn{2}{c}{ Priors } & \\
\hline Parameters & \multicolumn{2}{c}{ Prior Bounds } & Scale \\
\hline $\mathrm{H}_{2} \mathrm{O}$ & -12 & -2 & $\log$ \\
$\mathrm{CH}_{4}$ & -12 & -2 & $\log$ \\
$\mathrm{CO}$ & -12 & -2 & $\log$ \\
$\mathrm{CO}_{2}$ & -12 & -2 & $\log$ \\
$\mathrm{NH}_{3}$ & -12 & -2 & $\log$ \\
$T_{\text {term }}(\mathrm{K})$ & 400 & 2000 & $\operatorname{linear}$ \\
$P_{\text {clouds }}(\mathrm{Pa})$ & 6 & 1 & $\log$ \\
$R_{p}\left(R_{\text {jup }}\right)$ & 0.6 & 2.4 & $\operatorname{linear}$ \\
\hline $\mathrm{K}$ & -12 & -2 & $\log$ \\
$\mathrm{Na}$ & -12 & -2 & $\log$ \\
$P_{\text {mie }}(\mathrm{Pa})$ & 6 & 0 & $\log$ \\
$\chi_{\text {mie }}$ & -20 & -5 & $\log$ \\
Offset $[\mathrm{ppm}]$ & -5000 & 5000 & $\operatorname{linear}$ \\
\hline
\end{tabular}

Note. The top half of the table shows the parameters fitted for during the HST only retrieval and the lower half contains those that were during the fitting that included the VLT data.

\subsection{Atmospheric Modeling}

The retrieval of the transmission spectrum was performed using the publicly available retrieval suite TauREx 3 (Al-Refaie et al. 2019). ${ }^{5}$ For the star parameters and the planet mass, we used the values from Hellier et al. (2014). In our runs we assumed that WASP-96 $\mathrm{b}$ possesses a primary atmosphere with a ratio $\mathrm{He} / \mathrm{H}_{2}=0.17$ (i.e., solar abundance). To this, we added trace gases and included the molecular opacities from the ExoMol (Tennyson et al. 2016), HITRAN (Gordon et al. 2016), and HITEMP (Rothman \& Gordon 2014) databases for the following molecules: $\mathrm{H}_{2} \mathrm{O}$ (Polyansky et al. 2018), $\mathrm{CH}_{4}$ (Yurchenko et al. 2017), $\mathrm{CO}$ (Li et al. 2015), $\mathrm{CO}_{2}$ (Rothman et al. 2010) $\mathrm{NH}_{3}$ (Coles et al. 2019), K, and $\mathrm{Na}$ (Kramida et al. 2013). The line-broadened profiles for the resonance doublets of $\mathrm{Na}$ and $\mathrm{K}$ are computed using Allard et al. (2016) and Allard et al. (2019). For the clouds, we use gray opaque clouds and the Mie cloud model from Lee et al. (2013). On top of this, we also included collision-induced absorption (CIA) from $\mathrm{H}_{2}$ to $\mathrm{H}_{2}$ (Abel et al. 2011; Fletcher et al. 2018) and $\mathrm{H}_{2}-\mathrm{He}$ (Abel et al. 2012) as well as Rayleigh scattering for all molecules. We assumed isothermal and isochemical profiles throughout all our retrievals.

In our retrieval analysis, we used log uniform priors for all parameters as described in Table 3. Finally, we explored the parameter space using the nested sampling algorithm Multinest (Feroz et al. 2009) with 750 live points and an evidence tolerance of 0.5 .

\section{Results}

\subsection{Retrieval Results: HST Observation Only}

The top panel of Figure 5 shows the best-fit spectrum from our retrieval based on G102 and G141 data only. The best-fit model contains modulations that correspond to multiple absorption features of water, clearly indicating the presence of the molecule in the atmosphere. Our free Bayesian

\footnotetext{
https://github.com/ucl-exoplanets/TauREx3_public
}

retrieval analysis recovered a water abundance of $\log \left(\mathrm{H}_{2} \mathrm{O}\right)=$ $-3.08_{-1.81}^{+1.08}$, consistent with predictions from equilibrium chemistry models (Agúndez et al. 2012; Stock et al. 2018; Woitke et al. 2018). Assuming an isothermal temperature profile, our retrieval determines a temperature $T=609_{-120}^{+173} \mathrm{~K}$, which is lower than the equilibrium temperature of the planet and much lower than the temperature obtained by Nikolov et al. (2018). Simulations have demonstrated that the complexity of the terminator limb, which includes three-dimensional asymmetries in the chemical and thermal structure of the terminator region, often affects the absolute retrieved temperature and can have biases (Caldas et al. 2019; MacDonald et al. 2020; Pluriel et al. 2020a). A lower than expected temperature is often retrieved from HST WFC3 data (e.g., Skaf et al. 2020). Similarly, narrow wavelength coverage can result in wrong estimations of the atmospheric temperatures, which can be unstable if a single molecular band is probed (e.g., Rocchetto et al. 2016; Tsiaras et al. 2018; Pinhas et al. 2019). On the other hand, our analysis also shows a gray cloud top pressure at $\log \left(P_{\text {clouds }}\right)=4.39_{-1.21}^{+0.95}$, which seems to suggest a relatively clear atmosphere, confirming the findings of Nikolov et al. (2018). We did not fit for more complicated scattering models, as the HST wavelength range does not cover a sufficiently large wavelength range (in particular shorter wavelength) to constrain atmospheric scattering model (see Appendix A for results from fitting a more complicated scattering model). Due to the weak absorptions, especially compared to water, of other molecules in the wavelengths considered, we were not able to determine the molecular abundance of $\mathrm{NH}_{3}, \mathrm{CO}$, and $\mathrm{CH}_{4}$. For $\mathrm{NH}_{3}$, we were able to extract a $1 \sigma$ upper bound of $\log \left(\mathrm{NH}_{3}\right)_{\text {upper }}=-6.51$. The posterior distributions for this retrieval are shown in Figure 6.

\subsection{Offset between HST and VLT Observations}

The top panel of Figure 5 shows the HST spectra (G102 and G141) analyzed here. We also display the raw spectrum obtained with the VLT in Nikolov et al. (2018). With no correction, we can immediately observe that the two sets of spectra are not compatible. In particular, a significant offset between the the ground-based (orange) and space-based data (blue and dark blue) at around $0.8 \mu \mathrm{m}$. There are a number of potential sources for the differences seen here: variations in the stellar properties, instrument systematics, differences in the reduction pipelines, telluric corrections and the use of different orbital parameters or limb-darkening coefficients.

An imperfect correction of instrument systematics certainly has the potential to significantly alter the recovered transit depth, the best-fit models of the systematics, namely the orbital and long period ramps, are shown in Figure 7. The corrections applied to some exposures are greater than the offset seen between the HST and VLT data, which may explain the offset observed. However, the ramps seen in the G102 and G141 data are very different yet they have both been fitted such that the final data products are seemingly in good agreement. The observations had different exposure times (179.05 s; $156.70 \mathrm{~s})$, scan lengths $(2 ! .41 ; 3$ !' 60$)$ and scan rates $(0 !) 013 \mathrm{~s}^{-1}$; 0 ". $\left.022 \mathrm{~s}^{-1}\right)$, so different systematics are to be expected.

For both HST observations we fitted a linear long-term trend, in line with many previous studies. Guo et al. (2020) suspected, from visual inspection, that the trends seen in the WFC3 data of HD 97658b deviated from a linear fit. Thus, they experimented with a number of different trends (quadratic, exponential, 

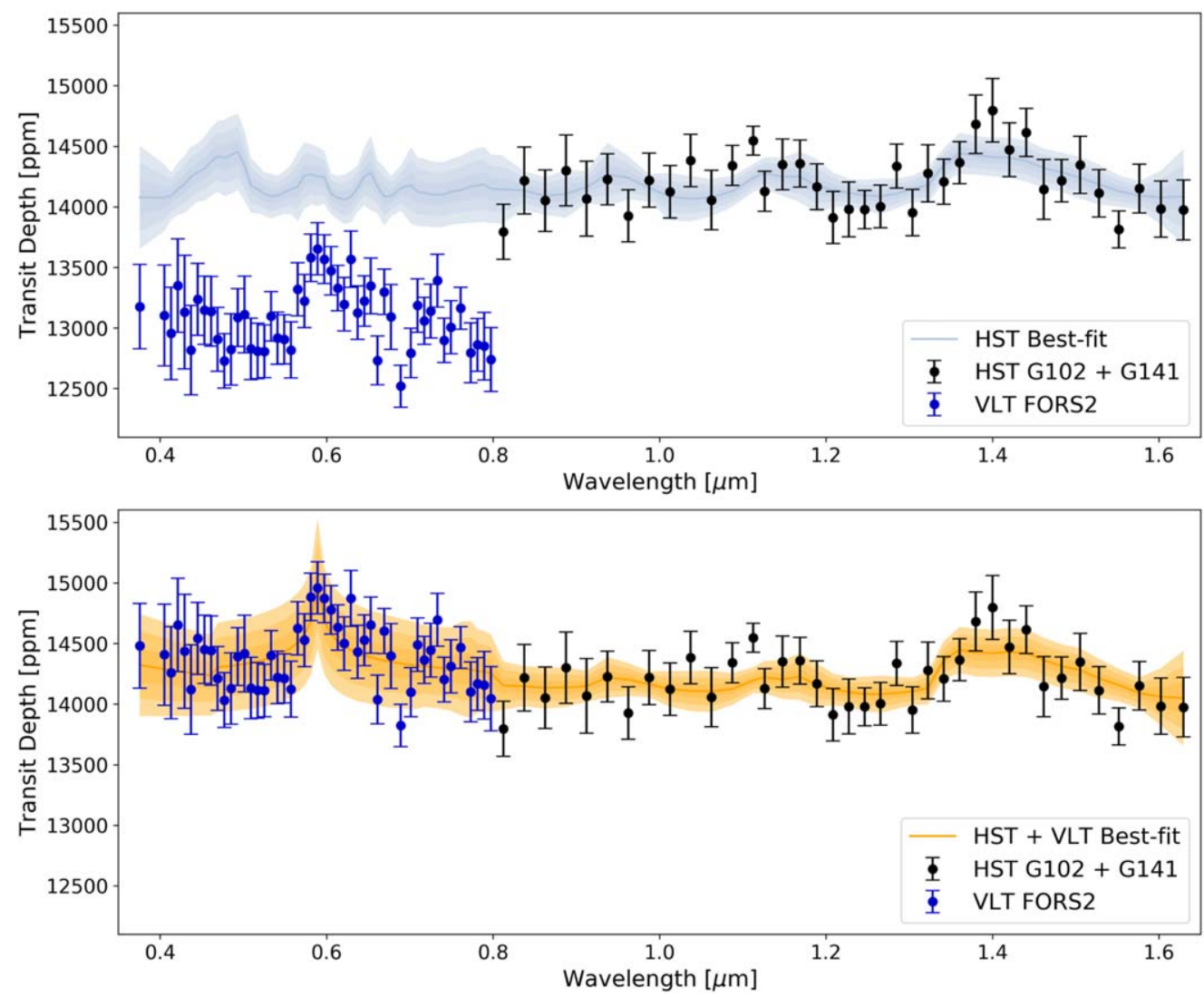

Figure 5. Transit depths derived from HST data (this work) with the addition of data from the VLT (Nikolov et al. 2018). A clear offset can be seen between the ground-based and space-based data sets (top panel). In both panels we overplot the best-fit spectrum for HST only (top, in blue) and HST+VLT after correction (bottom, in orange).

logarithmic) finding that, while the light-curve depths recovered from each observation were roughly consistent with one another, the chosen trend affected the transit depth recovered from the white light curves. They noted that the quadratic model produced the most uncertain depth as well as being the most discrepant between visits. Agol et al. (2010) also saw a bias when fitting Spitzer data with a quadratic trend. Here, visual examination of the raw light curves does not indicate that long-term trends are nonlinear, thus we do not explore different detrending modules.

In Nikolov et al. (2018), the authors noted that the derived depths from their two visits had marginal disagreement $(1.4 \sigma)$. Said difference was $720 \mathrm{ppm}$ but the authors noted that this level of variation is consistent with the photometric variability of the star, which is associated with active regions on its surface, of $920 \mathrm{ppm}$. The variation in the stellar flux could therefore be the cause of much of the offset seen here. We note that this could also be causing differences between the G102 and G141 observations though the derived depths are within $1 \sigma$. These were taken 10 days apart while the VLT data sets were acquired with a gap of 24 days.

The effect of combining different instruments is studied in the literature. Alexoudi et al. (2018) highlighted the importance of the using the correct orbital parameters for data covering visible wavelengths: otherwise slopes can be induced, or removed. Yip et al. (2020) highlight the danger of combining data from HST and the Spitzer Space Telescope, which are not sharing a common baseline and where the information redundancy for carbon-based species is limited. Despite this, studies often combine these data (e.g., Sing et al. 2016;
Pinhas et al. 2019). The combination of individual analyses from two different sets of observations may not necessarily agree with each other and should be approached with care.

The case we presented here is an obvious example of when the offset can be visually inspected but it is not the first study to introduce an offset to a data set. Bruno et al. (2020) showed that, for the active star WASP-52, stellar spots could create incoherent observations. The team combined transit data from HST/STIS, HST/WFC3, and Spitzer/IRAC of WASP-52 b and corrected for the offset in HST/WFC3 by accounting for the effect of stellar spots before retrieving the planet's atmospheric composition based on the corrected observations.

Kirk et al. (2019) explored the effect of stellar activity on the atmospheric retrieval of WASP-39 b when combining different data sets. In that study, the infrared data came from HST WFC3 G102 and G141, while the optical data sets were from the ground-based ACAM instrument on the $4.2 \mathrm{~m}$ William Herschel Telescope or from HST STIS, providing continuous coverage from 0.4 to $1.6 \mu \mathrm{m}$. They found no noticeable offset and little difference in the retrieved parameters when accounting for stellar activity, suggesting that, for WASP-39 b, the data sets could be compatible.

Other studies have applied, or fitted for, offsets without wavelength overlap. In a study of WASP-74 b with photometry from ground-based instruments along with HST/WFC3 and Spitzer/IRAC, Luque et al. (2020) fitted for an offset for the HST data, discovering a best-fit value of 434 or $615 \mathrm{ppm}$, depending upon the additional data used. Wilson et al. (2020) obtained FORS2 data of WASP-103 b and combined it with other ground-based data as well as data from HST and Spitzer. 


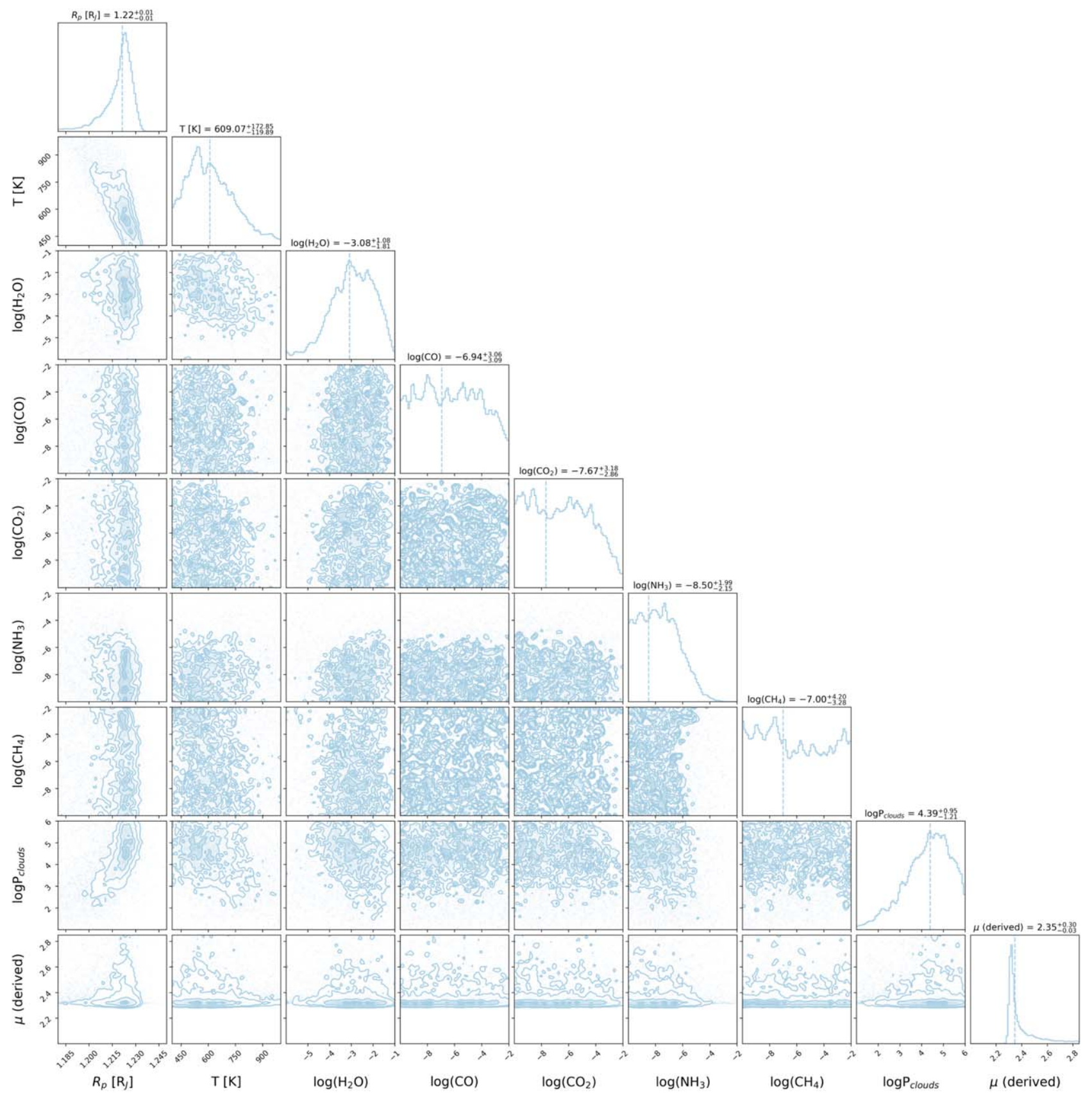

Figure 6. Posteriors distribution of different atmospheric parameters retrieved using HST data only (top of Figure 5).

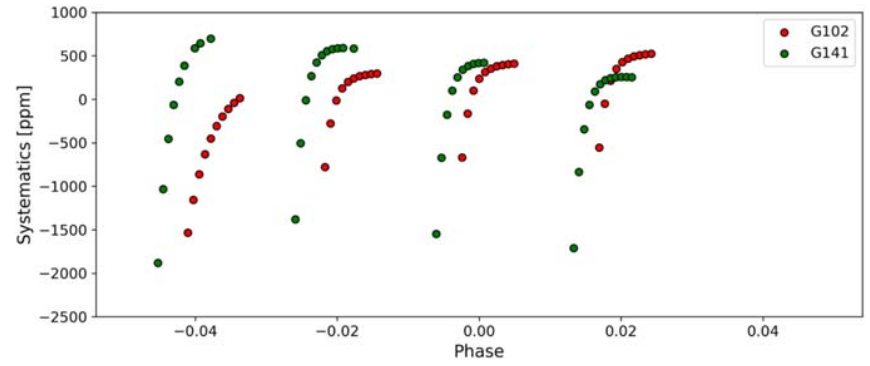

Figure 7. Best-fit systematics for the two HST observations analyzed here.

They applied a small offset to match the GMOS and FORS data before including an offset parameter in their retrievals to account for any further discrepancies. Meanwhile, in their study of HAT-P-12 b, Yan et al. (2020) attempted to fit for two offsets to improve their fitting of HST WFC3, HST STIS, and LBT data but found it did not lead to solutions that were statistically more valid. Finally, when fitting for an offset in the case of WASP-69 b between HST WFC3 and OSIRIS observations, a value of 479 or $618 \mathrm{ppm}$ was recovered by Murgas et al. (2020) with/without also fitting for a spot correction. They noted the cause could be biases due to instrument systematics, but that largest semiamplitude of the WASP photometry, $13 \mathrm{mmag}$, would result in a flux variation of $2.4 \%$, which could account for the $2.2 \%$ increase in flux between the observations.

However, there have been other instances in the literature where ground-based and space-based observations are combined and analyzed without wavelength overlap nor offset correction. For example, Danielski et al. (2014) combined 
observations from NASA Infrared Telescope Facility (IRTF)/ SpeX instrument and WFC3 observations on HD 189733b, many others followed similar trends (e.g., Bean et al. 2013; Mancini et al. 2013; Stevenson et al. 2016; Sotzen et al. 2020). While these data sets could be compatible, there is no guarantee and this should not be taken for granted.

In our case, without assigning a specific cause, we attempted to correct for the offset by fitting a single flat offset parameter in a combined retrieval. The parameter applies a shift to the entire VLT spectrum vertically to create a coherent observation with the HST data set. This choice of correction does not necessarily represent the complexity of the problem here and it is not guaranteed that the instrument systematics are wavelength-independent.

\subsection{Retrievals Results: HST + VLT Observations}

The best-fit spectrum is plotted in the bottom panel of Figure 5. Our combined retrieval unveiled an offset of $1198_{-102}^{+135} \mathrm{ppm}$ between the two instruments, as shown in the posterior distributions in Figure 8. The increased wavelength coverage (continuous coverage from 0.4 to $1.6 \mu \mathrm{m}$ ) allows us to fit for more complicated cloud models and probe the presence of two species $\left(\mathrm{H}_{2} \mathrm{O}\right.$ and $\left.\mathrm{Na}\right)$. The extension to the visible also helped to provide better constraint on the temperature on the terminator. The retrieved temperature $\left(T=954_{-195}^{+198} \mathrm{~K}\right)$ is close to the expected terminator temperature from a linear trend derived in Skaf et al. (2020), given the equilibrium temperature of the planet. An interesting potential explanation for the large temperatures difference between the $1700 \mathrm{~K}$ inferred from the VLT only data and the one retrieved from the HST only data could be that the signal from those two molecules comes from different regions of the terminator. Caldas et al. (2019), taking the example of $\mathrm{H}_{2} \mathrm{O}$ and $\mathrm{CO}$, predicted that some molecules could have a signal from the dayside part of the terminator region only or inversely the nightside part only.

The retrieved abundances for $\mathrm{H}_{2} \mathrm{O}$ and $\mathrm{Na}$ in our combined retrieval are within $2 \sigma$ agreement with the individual analysis reported in our work and Nikolov et al. (2018; see Table 4 for a comparison between retrieval scenarios). The consistency in the water abundances indicates that the water feature may be stable enough to be retrieved accurately with HST, even in the case of the low terminator temperature recovered in Section 3.1. Such a result is expected given the strong features of $\mathrm{H}_{2} \mathrm{O}$ in the WFC3 range and lack thereof in the visible.

Based on the retrieval result and the visually compatible observations in Figure 5, it may be tempting to conclude that the correction has been successful. However, we would like to emphasize here that this kind of correction is an ad hoc solution to the problem and does not contain any theoretical support. Any conclusion drawn from these kinds of combined observations should be treated lightly and comparisons to model fitting on single data sets should be made.

A retrieval study of 10 hot Jupiters by Pinhas et al. (2019) found that optical data played a significant role in ensuring that a reliable constraint on the abundances derived from infrared data could be placed. When combining optical and infrared data of HD 209458b from HST (STIS + G141), they found the constraints on water to be narrowed by a factor of 3 . However, the abundances retrieved in each case were drastically different: $\log \left(\mathrm{H}_{2} \mathrm{O}\right)_{\mathrm{WFC} 3}=-3.3_{-0.75}^{+0.80}$ and $\log \left(\mathrm{H}_{2} \mathrm{O}\right)_{\mathrm{STIS}+\mathrm{WFC} 3}=$ $-4.66_{-0.30}^{+0.39}$.

In contrast, the water abundances recovered here, with and without optical data, are in good agreement with one another (well within $1 \sigma$ ). This may well be due to the addition of the G102 grism, which has not been used to observe HD 209458b, highlighting that it has the capability to provide excellent constraints on the water abundance when combined with G141. Additionally, while here we focus on a case where groundbased and space-based instruments demonstrate an offset, such a discrepancy could also occur in space-based data sets from different instruments. Combining HST STIS, HST WFC3 G141, and Spitzer IRAC has become commonplace in the field (e.g., Sing et al. 2016). Given that there is no wavelength overlap in these studies, there is a risk of offsets occurring that could bias the results of subsequent atmospheric retrievals. For instances, the Spitzer IRAC bands cover spectral regions where carbon-bearing molecules such as $\mathrm{CH}_{4}, \mathrm{CO}$, and $\mathrm{CO}_{2}$ absorb, which could be biased when the instrument is not wellcalibrated, leading to wrong estimates of the $\mathrm{C} / \mathrm{O}$ ratio. The G102 grism remains an underutilized instrument for exoplanet spectroscopy but would offer extra confidence that the STIS and G141 data sets are compatible by providing wavelength overlap with both.

\subsection{TESS and Spitzer Transit Depths}

In addition to VLT observation, we have also explored the scenario when observations from Spitzer and TESS are added to the HST data. Figure 9 shows the TESS and Spitzer data plotted alongside the best-fit spectrum from the HST and corrected VLT retrieval from the previous section. The two Spitzer points $(3.6 \mu \mathrm{m}$ and $4.5 \mu \mathrm{m})$ are within $1 \sigma$ of the best-fit solution. Hence, it is possible that Spitzer is consistent with the other instruments. However, we remain cautious, and as there is no wavelength overlap for Spitzer, we cannot use the methodology employed for the correction of the VLT data. Additionally, given the size of the error bars on the Spitzer data, little additional spectral information would be gained. The TESS point, on the other hand, is about $2 \sigma$ away from the solution. Seven transits were observed with TESS and the depth of each of these is shown in Figure 10. While several of the individual observations are consistent with the HST + VLT best-fit (to $1 \sigma$ ), the weighted mean of these observations is larger than the model (orange bar). The average transit depth is larger than that obtained with HST, and the Spitzer points are seemingly consistent with the HST data. This provides further indications that the source of the offset seen between data sets may be caused by the reduction and analysis of the VLT observations. The transit depth data for all instruments is given in Table 6.

\subsection{Ephemeris Refinement}

We found that the observed HST and TESS transits were just outside the $1 \sigma$ literature ephemeris. Hence, we refined the period and reference midtransit time using the original ephemeris from Hellier et al. (2014), and the new data analyzed. We determined the ephemeris of WASP-96b to be $P=3.42525650 \pm$ 0.00000043 days and $T_{0}=2457665.84332 \pm 0.00014 \mathrm{BJD}_{\mathrm{TDB}}$, where $P$ is the planet's period, $T_{0}$ is the reference midtime of the transit and $\mathrm{BJD}_{\mathrm{TDB}}$ is the barycentric Julian date in the barycentric 


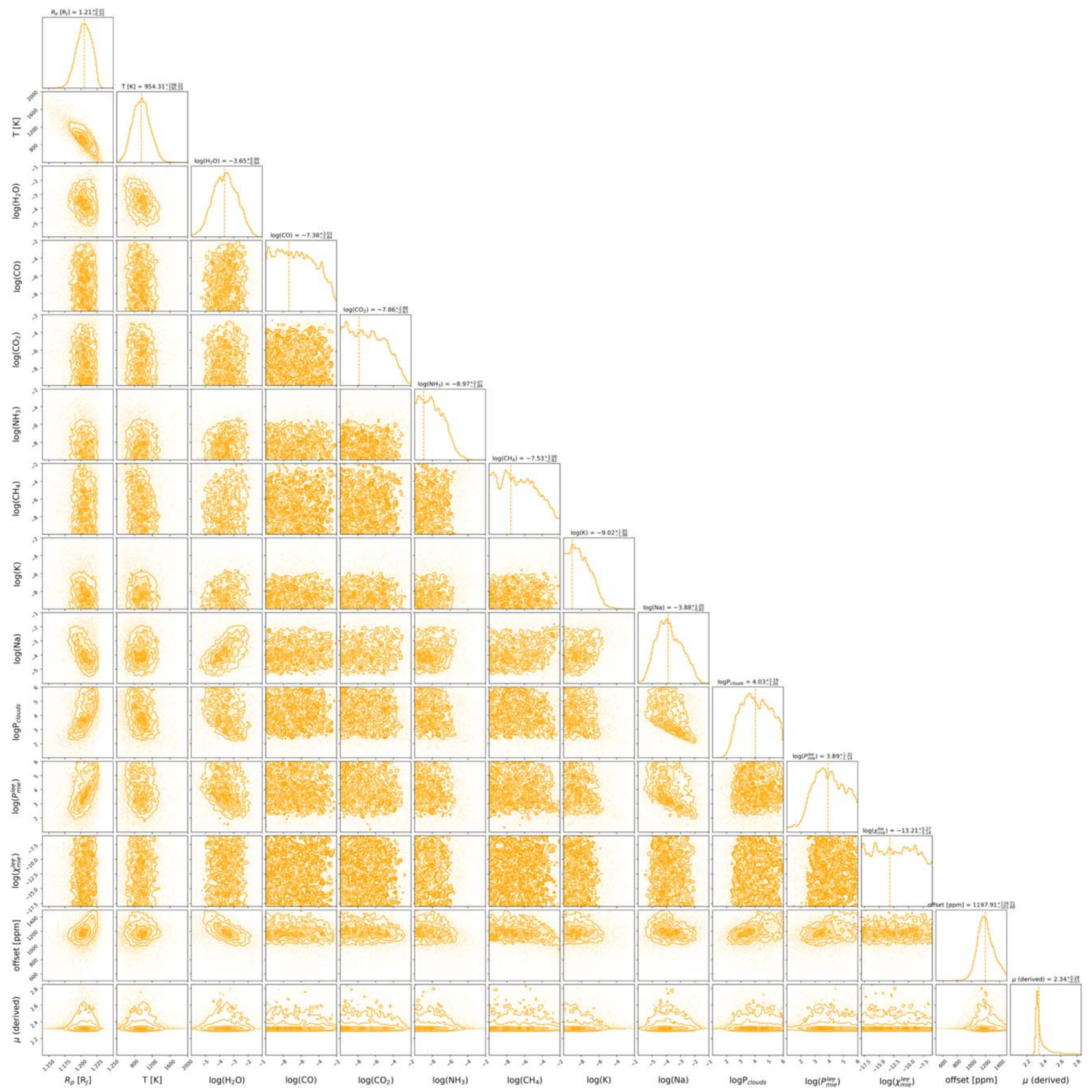

Figure 8. Posteriors distribution of different atmospheric parameters retrieved using both HST data and VLT data (after offset correction, bottom of Figure 5).

Table 4

Comparison between Different Retrieved Quantities for Three Different Scenarios (HST Only, VLT Only, and HST+VLT)

\begin{tabular}{lccc}
\hline \hline Parameter & HST & (Nikolov et al.) & HST+VLT \\
\hline $\log \left(\mathrm{H}_{2} \mathrm{O}\right)$ & $-3.08_{-1.81}^{+1.08}$ & $\mathrm{~N} / \mathrm{A}$ & $-3.65_{-0.94}^{+0.90}$ \\
$\log (\mathrm{Na})$ & $\mathrm{N} / \mathrm{A}$ & $-5.1_{-0.4}^{+0.6}$ & $-3.88_{-0.82}^{+1.05}$ \\
$R_{p}\left[R_{J}\right]$ & $1.22_{-0.01}^{+0.01}$ & $\mathrm{~N} / \mathrm{A}$ & $1.21_{-0.01}^{+0.01}$ \\
$\mathrm{~T}[\mathrm{~K}]$ & $609_{-120}^{+173}$ & $1710_{-200}^{+150}$ & $954_{-195}^{+198}$ \\
\hline
\end{tabular}

Note. Results from VLT alone are reproduced from Nikolov et al. (2018). We have omitted quantities that could not be constrained by any of the scenarios.

dynamical frame. Our derived period is $0.32 \mathrm{~s}$ shorter than that from Hellier et al. (2014) and we improved the precision of the period by a factor of 6 , thus reducing the current uncertainty on the transit time. The observed minus calculated plots are given in Figure 11 and all transit midtimes used for the fitting are listed in Table 5. We note that the Spitzer points are both seemingly poor fits to the trend, while the second HST observation (G141 grism) gives extremely tight bounds on the midtime despite the gaps within the light curve. We attempted an ephemeris fit without the HST observations and found little change in the period. TESS will soon reobserve WASP-96 b, which will allow for further refinement of its period. The midtimes have been uploaded to ExoClock ${ }^{6}$, an initiative to ensure transiting planets are regularly followed up, keeping their ephemeris up-to-date for the ESA Ariel mission (Tinetti et al. 2018; Edwards et al. 2019).

\footnotetext{
6 https://www.exoclock.space
} 


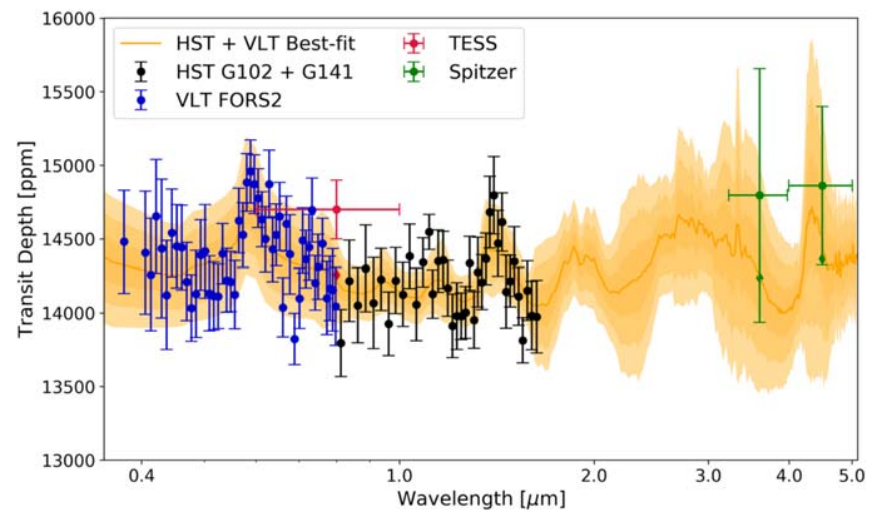

Figure 9. Best-fit model to the VLT and HST observations with data from TESS and Spitzer overplotted. The diamonds denote the transit depth of the model across these entire bands. The Spitzer data are within $1 \sigma$ of the retrieval model but the error bars are so high that little extra information could be gained by including them in the retrieval. Additionally, the risk of an offset is still present but undetectable given the lack of wavelength overlap.

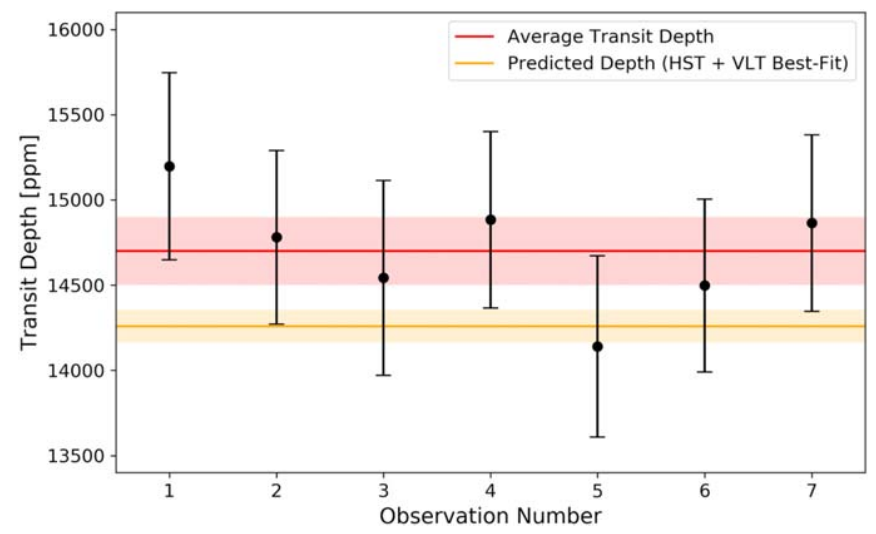

Figure 10. TESS transit depths from each individual observation and their weighted average. This can be seen to disagree by $2 \sigma$ compared to the expected depth from our HST + VLT retrieval.

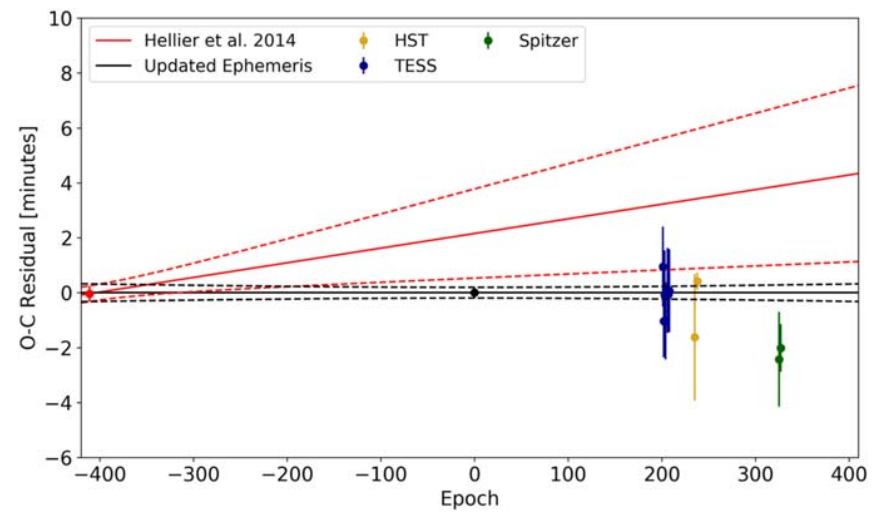

Figure 11. Observed minus calculated (O-C) midtransit times for WASP-96 b. Transit midtime measurements from this work are shown in gold (HST), blue (TESS) and green (Spitzer), while the $T_{0}$ value from Hellier et al. (2014) is in red. The black line denotes the new ephemeris of this work, with the dashed lines showing the associated $1 \sigma$ uncertainties and the black data poin indicating the updated $T_{0}$. For comparison, the previous literature ephemeris and their $1 \sigma$ uncertainties are given in red.

\subsection{WASP-96 b in Context}

Water appears to be ubiquitous in exoplanetary atmospheres. To understand the distribution in the abundance of this molecule, we compared the retrieved water (log) abundance
Table 5

Transit Midtimes Used to Refine the Ephemeris of Planets from This Study

\begin{tabular}{lll}
\hline \hline Epoch & \multicolumn{1}{c}{ Midtime $\left[\mathrm{BJD}_{\mathrm{TDB}}\right]$} & Reference \\
\hline-411.0 & $2456258.062876 \pm 0.0002$ & Hellier et al. (2014) \\
201.0 & $2458354.320536 \pm 0.000885$ & This Work (T) \\
202.0 & $2458357.744414 \pm 0.000859$ & This Work (T) \\
203.0 & $2458361.170328 \pm 0.000851$ & This Work (T) \\
204.0 & $2458364.594941 \pm 0.000814$ & This Work (T) \\
206.0 & $2458371.446218 \pm 0.000895$ & This Work (T) \\
207.0 & $2458374.871373 \pm 0.000854$ & This Work (T) \\
208.0 & $2458378.296724 \pm 0.000876$ & This Work (T) \\
235.0 & $2458470.777446 \pm 0.001133$ & This Work (H) \\
238.0 & $2458481.054632 \pm 0.000184$ & This Work (H) \\
325.0 & $2458779.050000 \pm 0.001200$ & This Work (S) \\
327.0 & $2458785.900800 \pm 0.000600$ & This Work (S) \\
\hline
\end{tabular}

T: TESS, H: Hubble, S: Spitzer

Note. Data that were originally in HJD time format were converted using the tool from Eastman et al. (2010).

$\left(\log \left(\mathrm{H}_{2} \mathrm{O}\right)\right.$ of exoplanets with similar sizes $\left( \pm 0.5 R_{J}\right)$ and masses $\left( \pm 0.2 M_{J}\right)$ against their respective equilibrium temperature (see the top panel of Figure 12) using data from Tsiaras et al. (2018), Pinhas et al. (2019), and Skaf et al. (2020). While these trends are not statistically significant yet, at temperatures of 1200-1400 K, planets appear to generally have a high water abundance. These planets are also closest in terms of size and mass to WASP-96 b (lower panel of Figure 12). An HST WFC3 study of Kepler-51 b and d, which have radii of $0.61 R_{J}$ and $0.84 R_{J}$, respectively, uncovered flat spectra for both, with no discernible atmospheric features (Libby-Roberts et al. 2020). However, these planets have noticeably cooler temperatures than WASP-96 b (400-550 K) and very low densities $\left(<0.07 \mathrm{~g} \mathrm{~cm}^{-3}\right)$. While the addition of our detection adds weight toward the tendency of having water rich atmosphere for this class of hot planets, more objects of a similar class are needed in order to statistically verify this claim.

\section{Conclusions}

WASP-96 b is one of many planets observed by both spacebased and ground-based instruments. Each instrument is sensitive to different chemical species and the combined wavelength spans from the optical to the near-infrared. In this investigation we detrended light curves obtained from WFC3/ HST and detected strong evidence for the presence of water, along with ruling out a large abundance of $\mathrm{NH}_{3}$, in the atmosphere of this hot Jupiter.

As we tried to combine our data with observations from Nikolov et al. (2018), we observed a large offset $\left(1197{ }_{-101}^{+134}\right.$ ppm) from the combined transmission spectrum. The inconsistency between them rendered any retrieval to be impossible without any correction. We thus fit for an offset parameter during our retrieval on the combined observation, in an attempt to correct for the discrepancy. The combined retrieval shows a consistent water and sodium abundance with analyses on individual instruments. It was also able to retrieve a consistent temperature with the equilibrium temperature of the planet.

Despite having a seemingly better aligned spectrum and better constrained result after the correction was made, we would like to point out that such a correction does not make the two observations compatible, and in fact there is no theoretical 

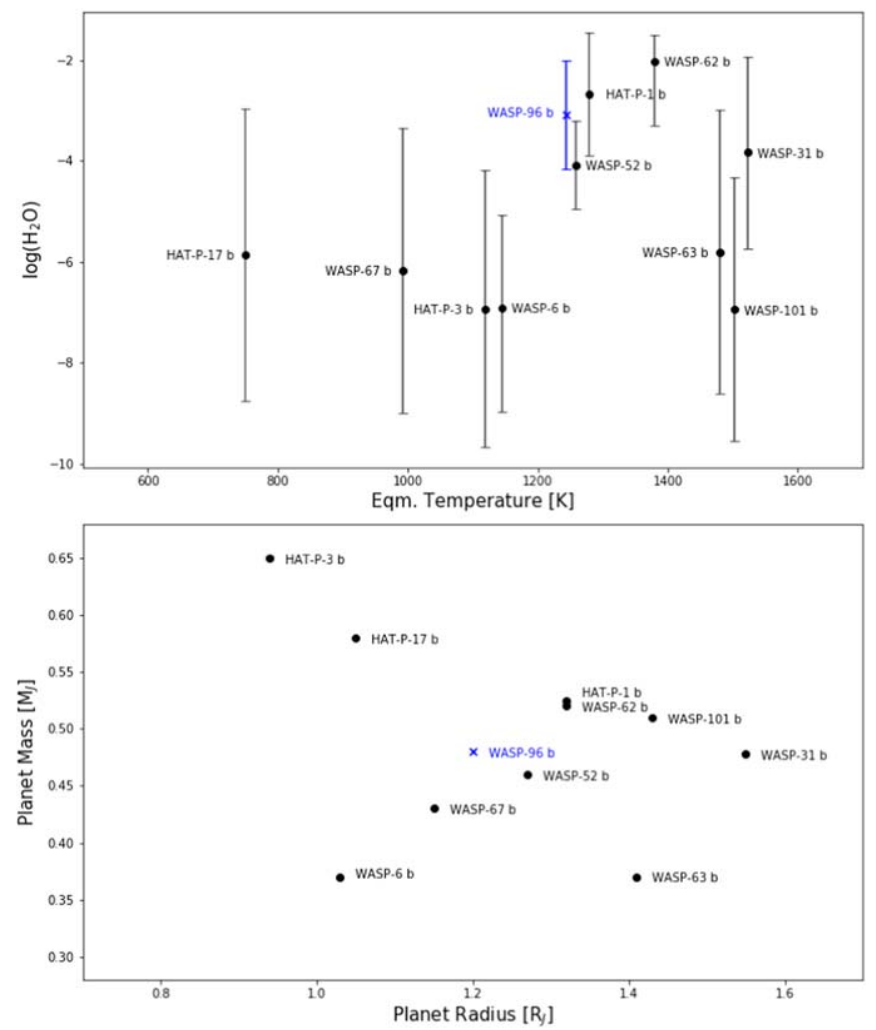

Figure 12. Comparing WASP-96 b with planets with a similar size and mass. Top: retrieved water $\log$ abundance of each planet against their respective equilibrium temperature. The water contents of WASP-96 b, WASP-52 b, and HAT-P-1 b are similar, as are their equilibrium temperatures. Bottom: massradius plot of exoplanets within $\pm 0.5 R_{J}$ and $\pm 0.2 M_{J}$ of WASP-96 b's radius and mass.

base for such a correction, therefore any conclusion should be taken with care.

The case of WASP-96 b served as an alarming example that compatibility between different instruments, be whether they are space-based or ground-based, should not be taken for granted. Seemingly consistent observations do not necessarily mean they are consistent. This is especially true when the observations do not overlap in wavelength.

The difficulty of confirming the compatibility between instruments will be mitigated by next-generation instrumentation such as JWST, Ariel (Tinetti et al. 2018), or Twinkle (Edwards et al. 2019). Their broad, simultaneous wavelength coverage will provide continuous coverage from optical to farinfrared at unprecedented resolution and signal-to-noise ratio, which could resolve the trouble of having to combine observations in exchange for broader wavelength coverage.

This project has received funding from the European Research Council (ERC) under the European Union's Horizon 2020 research and innovation program (grant agreement No. 758892, ExoAI) and under the European Union's Seventh
Framework Programme (FP7/2007-2013)/ ERC grant agreements No. 617119 (ExoLights). Furthermore, we acknowledge funding by the ASI grant No. 2018.22.HH.O and by the Science and Technology Funding Council (STFC) grants: ST/ K502406/1, ST/P000282/1, ST/P002153/1, ST/T001836/1, and ST/S002634/1.

This work is based upon observations with the NASA/ESA Hubble Space Telescope, obtained at the Space Telescope Science Institute (STScI) operated by AURA, Inc. The publicly available HST observations presented here were taken as part of proposal 15469 , led by Nikolay Nikolov. We are grateful to everyone involved in the creation of the proposals that led to these valuable HST and Spitzer data sets being made available to the community: Nikolay Nikolov, Gilda E. Ballester, Aarynn Carter, Drake Deming, Ben Drummond, Jonathan Fortney, Neale Gibson, Jayesh Goyal, Tiffany Kataria, Nathan J. Mayne, Thomas Mikal-Evans, David K. Sing, Jessica Spake, and Hannah Wakeford. These data were obtained from the Hubble Archive which is part of the Mikulski Archive for Space Telescopes. This paper also includes data collected by the TESS mission, which are publicly available from the Mikulski Archive for Space Telescopes (MAST) and produced by the Science Processing Operations Center (SPOC) at NASA Ames Research Center (Jenkins et al. 2016). This research effort made use of systematic error-corrected (PDC-SAP) photometry (Smith et al. 2012; Stumpe et al. 2012, 2014). Funding for the TESS mission is provided by NASA's Science Mission directorate. This work is also based in part on observations made with the Spitzer Space Telescope, which is operated by the Jet Propulsion Laboratory, California Institute of Technology, under a contract with NASA. These observations were taken as part of proposal 14255 , led by Nikolay Nikolov (Nikolov et al. 2019).

Software: Iraclis (Tsiaras et al. 2016b), TauREx3 (Al-Refaie et al. 2019), pylightcurve (Tsiaras et al. 2016a), ExoTETHyS (Morello et al. 2020), Astropy (Astropy Collaboration et al. 2018), h5py (Collette 2013), emcee (Foreman-Mackey et al. 2013), Matplotlib (Hunter 2007), Multinest (Feroz et al. 2009), corner (Foreman-Mackey 2016), Pandas (McKinney 2011), Numpy (Oliphant 2006), SciPy (Virtanen et al. 2020).

\section{Appendix A HST Spectrum with a More Complicated Model}

For better comparison with our results from VLT + HST observations (Figures 8 and 12), we applied the same atmospheric setup as VLT + HST retrieval to HST observations so as to understand the outcome from a more complicated model (see Figure 13). The result of the posterior distribution shows a similar outcome to our simpler model. The model was not able to constrain additional parameters, which is expected given the limited wavelength range from the instrument, hence we opted for simpler model to present in the main text. 


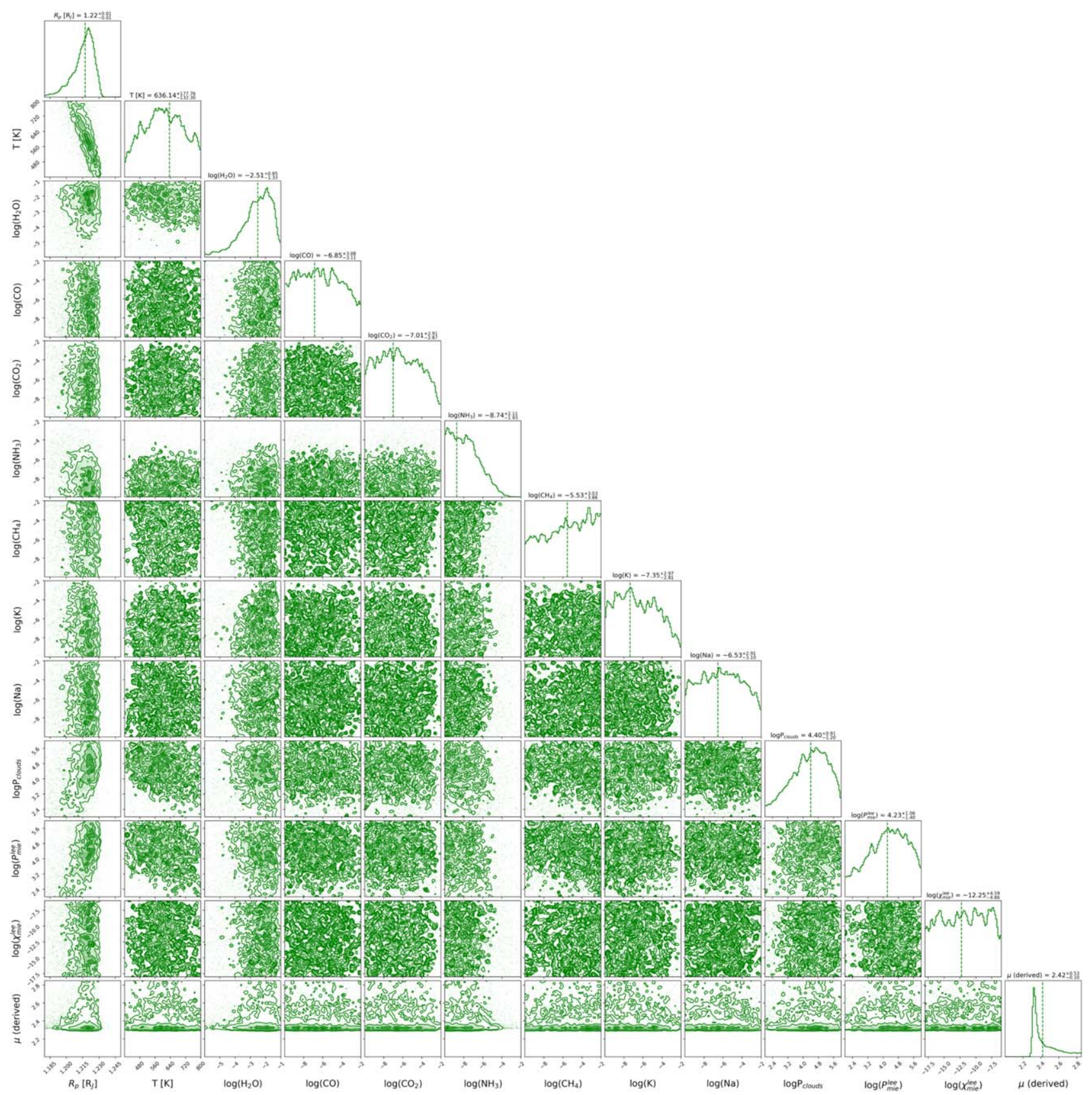

Figure 13. Posterior distribution of different atmospheric parameters retrieved using HST data with more complex atmospheric models. 


\section{Appendix B}

\section{Transit Depths Data from Multiple Instruments}

The transit depths derived using data from TESS, HST, Spitzer, and VLT (post offset correction) can be found in Table 6.

Table 6

The Transit Depths Derived Hhere for TESS, HST, and Spitzer along with the VLT Data, after Offset Correction

\begin{tabular}{|c|c|c|c|c|c|c|c|c|c|}
\hline $\begin{array}{l}\text { Wavelength } \\
(\mu \mathrm{m})\end{array}$ & $\begin{array}{c}\text { Transit } \\
\text { Depth (\%) }\end{array}$ & Error (\%) & $\begin{array}{l}\text { Bandwidth } \\
(\mu \mathrm{m})\end{array}$ & Instrument & $\begin{array}{c}\text { Wavelength } \\
\quad(\mu \mathrm{m})\end{array}$ & $\begin{array}{c}\text { Transit } \\
\text { Depth }(\%)\end{array}$ & Error $(\%)$ & $\begin{array}{c}\text { Bandwidth } \\
(\mu \mathrm{m})\end{array}$ & Instrument \\
\hline 0.37565 & 1.4065 & 0.0349 & 0.05130 & VLT FORS2 & 0.77330 & 1.3685 & 0.0247 & 0.00800 & VLT FORS2 \\
\hline 0.40530 & 1.3992 & 0.0417 & 0.00800 & VLT FORS2 & 0.78130 & 1.3750 & 0.0218 & 0.00800 & VLT FORS2 \\
\hline 0.41330 & 1.3844 & 0.0380 & 0.00800 & VLT FORS2 & 0.78930 & 1.3739 & 0.0279 & 0.00800 & VLT FORS2 \\
\hline 0.42130 & 1.4238 & 0.0386 & 0.00800 & VLT FORS2 & 0.79730 & 1.3628 & 0.0264 & 0.00800 & VLT FORS2 \\
\hline 0.42930 & 1.4019 & 0.0470 & 0.00800 & VLT FORS2 & 0.81250 & 1.3532 & 0.0179 & 0.00250 & HST G102 \\
\hline 0.43730 & 1.3705 & 0.0369 & 0.00800 & VLT FORS2 & 0.83750 & 1.3806 & 0.0239 & 0.00250 & HST G102 \\
\hline 0.44530 & 1.4125 & 0.0297 & 0.00800 & VLT FORS2 & 0.86250 & 1.3792 & 0.0220 & 0.00250 & HST G102 \\
\hline 0.45330 & 1.4035 & 0.0282 & 0.00800 & VLT FORS2 & 0.88750 & 1.3829 & 0.0313 & 0.00250 & HST G102 \\
\hline 0.46130 & 1.4026 & 0.0287 & 0.00800 & VLT FORS2 & 0.91250 & 1.3800 & 0.0311 & 0.00250 & HST G102 \\
\hline 0.46930 & 1.3796 & 0.0264 & 0.00800 & VLT FORS2 & 0.93750 & 1.3996 & 0.0189 & 0.00250 & HST G102 \\
\hline 0.47730 & 1.3617 & 0.0226 & 0.00800 & VLT FORS2 & 0.96250 & 1.3574 & 0.0205 & 0.00250 & HST G102 \\
\hline 0.48530 & 1.3712 & 0.0294 & 0.00800 & VLT FORS2 & 0.98750 & 1.3804 & 0.0215 & 0.00250 & HST G102 \\
\hline 0.49330 & 1.3976 & 0.0238 & 0.00800 & VLT FORS2 & 1.01250 & 1.3789 & 0.0199 & 0.00250 & HST G102 \\
\hline 0.50130 & 1.4001 & 0.0316 & 0.00800 & VLT FORS2 & 1.03750 & 1.4053 & 0.0216 & 0.00250 & HST G102 \\
\hline 0.50930 & 1.3716 & 0.0254 & 0.00800 & VLT FORS2 & 1.06250 & 1.3796 & 0.0234 & 0.00250 & HST G102 \\
\hline 0.51730 & 1.3698 & 0.0229 & 0.00800 & VLT FORS2 & 1.08750 & 1.3965 & 0.0140 & 0.00250 & HST G102 \\
\hline 0.52530 & 1.3696 & 0.0217 & 0.00800 & VLT FORS2 & 1.11250 & 1.4212 & 0.0111 & 0.00250 & HST G102 \\
\hline 0.53330 & 1.3985 & 0.0204 & 0.00800 & VLT FORS2 & 1.12625 & 1.3899 & 0.0162 & 0.02190 & HST G141 \\
\hline 0.54130 & 1.3805 & 0.0216 & 0.00800 & VLT FORS2 & 1.14775 & 1.4110 & 0.0200 & 0.02110 & HST G141 \\
\hline 0.54930 & 1.3796 & 0.0202 & 0.00800 & VLT FORS2 & 1.16860 & 1.4116 & 0.0210 & 0.02060 & HST G141 \\
\hline 0.55730 & 1.3707 & 0.0231 & 0.00800 & VLT FORS2 & 1.18880 & 1.3875 & 0.0181 & 0.01980 & HST G141 \\
\hline 0.56530 & 1.4208 & 0.0219 & 0.00800 & VLT FORS2 & 1.20835 & 1.3664 & 0.0217 & 0.01930 & HST G141 \\
\hline 0.57330 & 1.4111 & 0.0218 & 0.00800 & VLT FORS2 & 1.22750 & 1.3811 & 0.0210 & 0.01900 & HST G141 \\
\hline 0.58130 & 1.4468 & 0.0196 & 0.00800 & VLT FORS2 & 1.24645 & 1.3730 & 0.0152 & 0.01890 & HST G141 \\
\hline 0.58930 & 1.4542 & 0.0215 & 0.00800 & VLT FORS2 & 1.26550 & 1.3809 & 0.0185 & 0.01920 & HST G141 \\
\hline 0.59730 & 1.4456 & 0.0200 & 0.00800 & VLT FORS2 & 1.28475 & 1.4164 & 0.0185 & 0.01930 & HST G141 \\
\hline 0.60530 & 1.4361 & 0.0200 & 0.00800 & VLT FORS2 & 1.30380 & 1.3804 & 0.0191 & 0.01880 & HST G141 \\
\hline 0.61330 & 1.4217 & 0.0187 & 0.00800 & VLT FORS2 & 1.32260 & 1.4031 & 0.0229 & 0.01880 & HST G141 \\
\hline 0.62130 & 1.4084 & 0.0221 & 0.00800 & VLT FORS2 & 1.34145 & 1.3927 & 0.0180 & 0.01890 & HST G141 \\
\hline 0.62930 & 1.4456 & 0.0231 & 0.00800 & VLT FORS2 & 1.36050 & 1.4132 & 0.0170 & 0.01920 & HST G141 \\
\hline 0.63730 & 1.4015 & 0.0220 & 0.00800 & VLT FORS2 & 1.38005 & 1.4429 & 0.0238 & 0.01990 & HST G141 \\
\hline 0.64530 & 1.4111 & 0.0209 & 0.00800 & VLT FORS2 & 1.40000 & 1.4585 & 0.0241 & 0.02000 & HST G141 \\
\hline 0.65330 & 1.4236 & 0.0231 & 0.00800 & VLT FORS2 & 1.42015 & 1.4273 & 0.0206 & 0.02030 & HST G141 \\
\hline 0.66130 & 1.3621 & 0.0201 & 0.00800 & VLT FORS2 & 1.44060 & 1.4345 & 0.0191 & 0.02060 & HST G141 \\
\hline 0.66930 & 1.4187 & 0.0187 & 0.00800 & VLT FORS2 & 1.46150 & 1.3797 & 0.0227 & 0.02120 & HST G141 \\
\hline 0.67730 & 1.3980 & 0.0268 & 0.00800 & VLT FORS2 & 1.48310 & 1.4007 & 0.0183 & 0.02200 & HST G141 \\
\hline 0.68930 & 1.3408 & 0.0175 & 0.01600 & VLT FORS2 & 1.50530 & 1.4038 & 0.0218 & 0.02240 & HST G141 \\
\hline 0.70130 & 1.3682 & 0.0204 & 0.00800 & VLT FORS2 & 1.52800 & 1.3887 & 0.0190 & 0.02300 & HST G141 \\
\hline 0.70930 & 1.4074 & 0.0220 & 0.00800 & VLT FORS2 & 1.55155 & 1.3661 & 0.0154 & 0.02410 & HST G141 \\
\hline 0.71730 & 1.3948 & 0.0194 & 0.00800 & VLT FORS2 & 1.57625 & 1.3945 & 0.0170 & 0.02530 & HST G141 \\
\hline 0.72530 & 1.4029 & 0.0222 & 0.00800 & VLT FORS2 & 1.60210 & 1.3796 & 0.0235 & 0.02640 & HST G141 \\
\hline 0.73330 & 1.4280 & 0.0218 & 0.00800 & VLT FORS2 & 1.62945 & 1.3791 & 0.0217 & 0.02830 & HST G141 \\
\hline 0.74130 & 1.3787 & 0.0184 & 0.00800 & VLT FORS2 & 0.8 & 1.3803 & 0.0346 & 0.4 & TESS \\
\hline 0.74930 & 1.3896 & 0.0219 & 0.00800 & VLT FORS2 & 3.6 & 1.4796 & 0.0860 & 0.75 & Spitzer IRAC \\
\hline 0.76130 & 1.4052 & 0.0172 & 0.01600 & VLT FORS2 & 4.5 & 1.4861 & 0.0538 & 1.015 & Spitzer IRAC \\
\hline
\end{tabular}




\section{ORCID iDs}

Kai Hou Yip (ㄱ https://orcid.org/0000-0002-9616-1524 Quentin Changeat (ib https://orcid.org/0000-0001-6516-4493

Billy Edwards 누 https://orcid.org/0000-0002-5494-3237 Mario Morvan (ํ) https://orcid.org/0000-0001-8587-2112 Katy L. Chubb (1) https://orcid.org/0000-0002-4552-4559 Angelos Tsiaras 는 https://orcid.org/0000-0003-3840-1793 Ingo P. Waldmann (1) https://orcid.org/0000-0002-4205-5267 Giovanna Tinetti [i] https://orcid.org/0000-0001-6058-6654

\section{References}

Abel, M., Frommhold, L., Li, X., \& Hunt, K. L. 2011, JPCA, 115, 6805 Abel, M., Frommhold, L., Li, X., \& Hunt, K. L. 2012, JChPh, 136, 044319 Agol, E., Cowan, N. B., Knutson, H. A., et al. 2010, ApJ, 721, 1861 Agúndez, M., Venot, O., Iro, N., et al. 2012, A\&A, 548, A73 Alexoudi, X., Mallonn, M., von Essen, C., et al. 2018, A\&A, 620, A142 Allard, N. F., Spiegelman, F., \& Kielkopf, J. F. 2016, A\&A, 589, A21 Allard, N. F., Spiegelman, F., Leininger, T., \& Mollière, P. 2019, A\&A, $628, \mathrm{~A} 120$

Al-Refaie, A. F., Changeat, Q., Waldmann, I. P., \& Tinetti, G. 2019, arXiv: 1912.07759

Barman, T. S. 2008, ApJL, 676, L61

Barman, T. S., Konopacky, Q. M., Macintosh, B., \& Marois, C. 2015, ApJ, 804,61

Bean, J. L., Désert, J.-M., Seifahrt, A., et al. 2013, ApJ, 771, 108

Bourrier, V., Ehrenreich, D., Lendl, M., et al. 2020, A\&A, 635, A205

Bruno, G., Lewis, N. K., Alam, M. K., et al. 2020, MNRAS, 491, 5361

Caldas, A., Leconte, J., Selsis, F., et al. 2019, A\&A, 623, A161

Charbonneau, D., Brown, T. M., Noyes, R. W., \& Gilliland, R. L. 2002, ApJ, 568,377

Claret, A., Hauschildt, P. H., \& Witte, S. 2012, A\&A, 546, A14

Claret, A., Hauschildt, P. H., \& Witte, S. 2013, A\&A, 552, A16

Coles, P. A., Yurchenko, S. N., \& Tennyson, J. 2019, MNRAS, 490, 4638

Collette, A. 2013, Python and HDF5 (Sebastopol, CA: O'Reilly Media Inc.)

Danielski, C., Deroo, P., Waldmann, I. P., et al. 2014, ApJ, 785, 35

Eastman, J., Siverd, R., \& Gaudi, B. S. 2010, PASP, 122, 935

Edwards, B., Changeat, Q., Baeyens, R., et al. 2020a, AJ, 160, 8

Edwards, B., Changeat, Q., Yip, K. H., et al. 2020b, MNRAS, in press

Edwards, B., Mugnai, L., Tinetti, G., Pascale, E., \& Sarkar, S. 2019, AJ, 157,242

Edwards, B., Rice, M., Zingales, T., et al. 2019, ExA, 47, 29

Ehrenreich, D., Bonfils, X., Lovis, C., et al. 2014, A\&A, 570, A89

Ehrenreich, D., Lovis, C., Allart, R., et al. 2020, Natur, 580, 597

Feroz, F., Hobson, M. P., \& Bridges, M. 2009, MNRAS, 398, 1601

Fisher, C., \& Heng, K. 2018, MNRAS, 481, 4698

Fletcher, L. N., Gustafsson, M., \& Orton, G. S. 2018, ApJS, 235, 24

Foreman-Mackey, D. 2016, JOSS, 1, 24

Foreman-Mackey, D., Hogg, D. W., Lang, D., \& Goodman, J. 2013, PASP, 125,306

Fossati, L., Haswell, C. A., Froning, C. S., et al. 2010, ApJL, 714, L222

Gordon, I., Rothman, L. S., Wilzewski, J. S., et al. 2016, AAS/DPS Meeting, $48,421.13$

Guo, X., Crossfield, I. J. M., Dragomir, D., et al. 2020, AJ, 159, 239

Haynes, K., Mandell, A. M., Madhusudhan, N., Deming, D., \& Knutson, H. 2015, ApJ, 806, 146

Hellier, C., Anderson, D. R., Cameron, A. C., et al. 2014, MNRAS, 440, 1982 Hunter, J. D. 2007, CSE, 9, 90

Iyer, A. R., Swain, M. R., Zellem, R. T., et al. 2016, ApJ, 823, 109

Jenkins, J. M., Twicken, J. D., McCauliff, S., et al. 2016, Proc. SPIE, 9913 , 99133E

Kirk, J., López-Morales, M., Wheatley, P. J., et al. 2019, AJ, 158, 144

Kramida, A., Ralchenko, Y., \& Reader, J. 2013, NIST Atomic Spectra Database-Version 5, http://www.nist.gov/pml/data/asd.cfm

Gravity Collaboration, Lacour, S., Nowak, M., et al. 2019, A\&A, 623, L11

Lee, J.-M., Heng, K., \& Irwin, P. G. J. 2013, ApJ, 778, 97
Li, G., Gordon, I. E., Rothman, L. S., et al. 2015, ApJS, 216, 15

Libby-Roberts, J. E., Berta-Thompson, Z. K., Désert, J.-M., et al. 2020, AJ, 159,57

Linsky, J. L., Yang, H., France, K., et al. 2010, ApJ, 717, 1291

Luque, R., Casasayas-Barris, N., Parviainen, H., et al. 2020, A\&A, 642, A50

MacDonald, R. J., Goyal, J. M., \& Lewis, N. K. 2020, ApJL, 893, L43

MacDonald, R. J., \& Madhusudhan, N. 2017, ApJL, 850, L15

Macintosh, B., Graham, J. R., Barman, T., et al. 2015, Sci, 350, 64

Mancini, L., Ciceri, S., Chen, G., et al. 2013, MNRAS, 436, 2

Mandell, A. M., Haynes, K., Sinukoff, E., et al. 2013, ApJ, 779, 128

McKinney, W. 2011, in Proc. 9th Python in Scientific Conf., ed. S. van der Walt \& J. Millman (Austin, TX: SciPy), 56

Merritt, S. R., Gibson, N. P., Nugroho, S. K., et al. 2020, A\&A, 636, A117

Mikal-Evans, T., Sing, D. K., Kataria, T., et al. 2020, MNRAS, 496, 1638

Morello, G., Claret, A., Martin-Lagarde, M., et al. 2020, AJ, 159, 75

Morvan, M., Nikolaou, N., Tsiaras, A., \& Waldmann, I. P. 2020, AJ, 159, 109

Murgas, F., Chen, G., Nortmann, L., Pallé, E., \& Nowak, G. 2020, A\&A, 641, A158

Nikolov, N., Sing, D., Fortney, J., et al. 2019, Characterizing a New Prototype Saturn-mass Exoplanet with the Clearest Atmosphere Yet, Spitzer Proposal, 14255

Nikolov, N., Sing, D. K., Fortney, J. J., et al. 2018, Natur, 557, 526

Oliphant, T. E. 2006, A Guide to NumPy, Vol. 1 (Spanish Fork, UT: Trelgol Publishing)

Pinhas, A., Madhusudhan, N., Gandhi, S., \& MacDonald, R. 2019, MNRAS, 482, 1485

Pluriel, W., Whiteford, N., Edwards, B., et al. 2020b, AJ, 160, 112

Pluriel, W., Zingales, T., Leconte, J., \& Parmentier, V. 2020a, A\&A, 636, A66

Polyansky, O. L., Kyuberis, A. A., Zobov, N. F., et al. 2018, MNRAS, 480, 2597

Astropy Collaboration, Price-Whelan, A. M., Sipőcz, B. M., et al. 2018, AJ, 156,123

Redfield, S., Endl, M., Cochran, W. D., \& Koesterke, L. 2008, ApJL, 673, L87

Ricker, G. R., Winn, J. N., Vanderspek, R., et al. 2014, Proc. SPIE, 9143 , 914320

Rocchetto, M., Waldmann, I. P., Venot, O., Lagage, P. O., \& Tinetti, G. 2016, ApJ, 833, 120

Rothman, L., Gordon, I., Barber, R., et al. 2010, JQSRT, 111, 2139

Rothman, L. S., \& Gordon, I. E. 2014, Status of the HITRAN and HITEMP Databases, Zenodo, doi:10.5281/zenodo. 11207

Sing, D. K., Fortney, J. J., Nikolov, N., et al. 2016, Natur, 529, 59

Skaf, N., Fabienne Bieger, M., Edwards, B., et al. 2020, AJ, 160, 109

Smith, J. C., Stumpe, M. C., Van Cleve, J. E., et al. 2012, PASP, 124, 1000

Sotzen, K. S., Stevenson, K. B., Sing, D. K., et al. 2020, AJ, 159, 5

Stevenson, K. B., Bean, J. L., Seifahrt, A., et al. 2016, ApJ, 817, 141

Stock, J. W., Kitzmann, D., Patzer, A. B. C., \& Sedlmayr, E. 2018, MNRAS, 479,865

Stumpe, M. C., Smith, J. C., Catanzarite, J. H., et al. 2014, PASP, 126, 100

Stumpe, M. C., Smith, J. C., Van Cleve, J. E., et al. 2012, PASP, 124, 985

Swain, M. R., Tinetti, G., Vasisht, G., et al. 2009, ApJ, 704, 1616

Tennyson, J., Yurchenko, S. N., Al-Refaie, A. F., et al. 2016, JMoSp, 327, 73

Tinetti, G., Drossart, P., Eccleston, P., et al. 2018, ExA, 46, 135

Tinetti, G., Vidal-Madjar, A., Liang, M.-C., et al. 2007, Natur, 448, 169

Tsiaras, A., Waldmann, I., Rocchetto, M., et al. 2016a, pylightcurve: Exoplanet Lightcurve Model, Astrophysics Source Code Library, ascl:1612.018

Tsiaras, A., Waldmann, I. P., Rocchetto, M., et al. 2016b, ApJ, 832, 202

Tsiaras, A., Waldmann, I. P., Tinetti, G., Tennyson, J., \& Yurchenko, S. N. 2019, NatAs, 3, 1086

Tsiaras, A., Waldmann, I. P., Zingales, T., et al. 2018, AJ, 155, 156

Vidal-Madjar, A., Désert, J.-M., Lecavelier des Etangs, A., et al. 2004, ApJL, 604, L69

Virtanen, P., Gommers, R., Oliphant, T. E., et al. 2020, NatMe, 17, 261

Wakeford, H. R., Sing, D. K., Deming, D., et al. 2018, AJ, 155, 29

Wilson, J., Gibson, N. P., Nikolov, N., et al. 2020, MNRAS, 497, 5155

Woitke, P., Helling, C., Hunter, G. H., et al. 2018, A\&A, 614, A

Yan, F., Espinoza, N., Molaverdikhani, K., et al. 2020, A\&A, 642, A98

Yip, K. H., Waldmann, I. P., Tsiaras, A., \& Tinetti, G. 2020, AJ, 160, 171

Yurchenko, S. N., Amundsen, D. S., Tennyson, J., \& Waldmann, I. P. 2017, A\&A, 605, A95 